\title{
Oscillation mode linewidths of main-sequence and subgiant stars observed by Kepler
}

\author{
T. Appourchaux ${ }^{1,2}$, O. Benomar ${ }^{3}$, M. Gruberbauer ${ }^{4}$, W. J. Chaplin ${ }^{5}$, R. A. García ${ }^{6}$, R. Handberg ${ }^{7}$, G. A. Verner ${ }^{6}$, \\ H. M. Antia ${ }^{8}$, T. L. Campante ${ }^{7,9}$, G. R. Davies ${ }^{6}$, S. Deheuvels ${ }^{10}$, S. Hekker ${ }^{11,5}$, R. Howe ${ }^{5}$, D. Salabert ${ }^{12}$, \\ T. R. Bedding ${ }^{3}$, T. R. White ${ }^{3}$, G. Houdek ${ }^{13}$, V. Silva Aguirre ${ }^{14}$, Y. P. Elsworth ${ }^{5}$, J. Van Cleve ${ }^{15}$, \\ B. D. Clarke ${ }^{15}$, J. R. Hall ${ }^{16}$, and H. Kjeldsen ${ }^{7}$ \\ ${ }^{1}$ Université Paris-Sud, Institut d'Astrophysique Spatiale, UMR8617, CNRS, Bâtiment 121, 91405 Orsay Cedex, France \\ e-mail: thierry.appourchaux@ias.u-psud.fr \\ 2 Kavli Institute for Theoretical Physics, University of California, Santa Barbara, CA 93106-4030, USA \\ 3 Sydney Institute for Astronomy (SIfA), School of Physics, University of Sydney, New South Wales 2006, Australia \\ ${ }^{4}$ Department of Astronomy \& Physics, Saint Mary's University, Halifax, NS B3H 3C3, Canada \\ 5 School of Physics and Astronomy, University of Birmingham, Edgbaston, Birmingham B15 2TT, UK \\ ${ }^{6}$ Laboratoire AIM, CEA/DSM-CNRS-Université Paris Diderot, IRFU/SAp, Centre de Saclay, 91191 Gif-sur-Yvette Cedex, France \\ 7 Department of Physics and Astronomy, Aarhus University, Ny Munkegade 120, 8 Aarhus C, Denmark \\ 8 Tata Institute of Fundamental Research, Homi Bhabha Road, Mumbai 45, India \\ 9 Centro de Astrofísica, DFA-Faculdade de Ciências, Universidade do Porto, Rua das Estrelas, 4150-762 Porto, Portugal \\ 10 Department of Astronomy, Yale University, PO Box 208101, New Haven CT 06520-8101, USA \\ 11 Astronomical Institute "Anton Pannekoek", University of Amsterdam, Science Park 904, 1098 XH Amsterdam, The Netherlands \\ 12 Université de Nice Sophia-Antipolis, CNRS UMR 6202, Observatoire de la Côte d'Azur, BP 4229, 06304 Nice Cedex 4, France \\ 13 Institute of Astronomy, University of Vienna, 1180 Vienna, Austria \\ 14 Max Planck Institute for Astrophysics, Karl-Schwarzschild-Str. 1, 85748 Garching bei München, Germany \\ 15 SETI Institute/NASA Ames Research Center, Moffett Field, CA 94035, USA \\ 16 Orbital Sciences Corporation/NASA Ames Research Center, Moffett Field, CA 94035, USA
}

Received 22 November 2011 / Accepted 13 December 2011

\section{ABSTRACT}

Context. Solar-like oscillations have been observed by Kepler and CoRoT in several solar-type stars.

Aims. We study the variations in the stellar p-mode linewidth as a function of effective temperature.

Methods. We study a time series of nine months of Kepler data. We analyse the power spectra of 42 cool main-sequence stars and subgiants using both maximum likelihood estimators and Bayesian estimators to recover individual mode characteristics such as frequencies, linewidths, and mode heights.

Results. We report on the mode linewidth at both maximum power and maximum mode height for these 42 stars as a function of effective temperature.

Conclusions. We show that the mode linewidth at either maximum mode height or maximum amplitude follows a scaling relation with effective temperature, which is a combination of a power law and a lower bound. The typical power-law index is about 13 for the linewidth derived from the maximum mode height, and about 16 for the linewidth derived from the maximum amplitude, while the lower bound is about $0.3 \mu \mathrm{Hz}$ and $0.7 \mu \mathrm{Hz}$, respectively. We stress that this scaling relation is only valid for cool main-sequence stars and subgiants, and does not have any predictive power outside the temperature range of these stars.

Key words. methods: data analysis - asteroseismology - stars: solar-type

\section{Introduction}

Stellar physics faces a revolution following the great wealth of asteroseismic data made available by space missions such as CoRoT (Baglin 2006) and Kepler (Borucki et al. 2009). Long observations of solar-like pulsators corresponding to main sequence stars, subgiants, and red giants were performed over more than six months by CoRoT (Baudin et al. 2011a,b, and references therein). The Kepler mission now provides a larger sample of stars observed over longer time intervals (Chaplin et al. 2011).

The study of oscillation mode physics (mode height, linewidth, and amplitude) provides information about the excitation and damping mechanisms related to the physics of convection and stellar atmospheres (Samadi 2009). Houdek et al. (1999) theoretically derived stellar mode linewidths as a function of stellar mass and age. They found that stellar mode linewidths would correspond either to a depression or plateau close to the maximum of mode height. This depression was first detected in the solar p-mode linewidths by Fröhlich et al. (1995) and is caused by a resonance between the thermal adjustment time of the superadiabatic boundary layer and the mode frequency (Balmforth 1992). The frequency location of the maximum of the mode height is in turn related to the Mach number $\left(\mathcal{M}_{a}\right)$, the ratio of convective velocity to the sound speed (Belkacem et al. 2011). The convective flux giving the maximum mode amplitude is also related to $\mathcal{M}_{a}$ to the power of three (e.g. Belkacem et al. 2011; Houdek et al. 1999). It is therefore interesting to study 
how the mode linewidth is related to the frequency of maximum amplitude/mode height for several different stars.

Statistical studies over a large number of stars have been performed to validate the scaling relation derived for the amplitude of stellar oscillations by Kjeldsen \& Bedding (1995) and revised by Kjeldsen \& Bedding (2011). Scaling relations for mode linewidth were proposed by Chaplin et al. (2009) and Baudin et al. (2011a) based on the stellar effective temperature.

Chaplin et al. (2009) proposed a scaling relation with linewidth that is proportional to $T_{\text {eff }}^{4}$ based on several groundbased observations. Using CoRoT observations, Baudin et al. (2011b) measured linewidths for a sample of solar-like pulsators and found a scaling relation proportional to $T_{\mathrm{eff}}^{16}$.

Owing to the ability to perform longer observations of stars with Kepler, the measurement of mode linewidth becomes easier and more reliable. In this paper, we analyse Kepler observations of a larger stellar sample than of Baudin et al. (2011a), to derive a new relation between mode linewidth and $T_{\text {eff }}$.

\section{Data analysis}

\subsection{Time series and power spectra}

Kepler observations are obtained in two different operating modes: long cadence (LC) and short cadence (SC) (Gilliland et al. 2010; Jenkins et al. 2010). Our analysis here is based on SC data. For the brightest stars (down to Kepler magnitude, $K p \approx 12$ ), SC observations can be obtained for a limited number of stars (up to 512 at any given time) with a faster sampling rate of $58.84876 \mathrm{~s}$ (Nyquist frequency of $\sim 8.5 \mathrm{mHz}$ ), allowing for more precise transit timing. The time series were corrected for outliers, occasional jumps, and drifts (see García et al. 2011), and the levels between the quarters were normalized. Finally, the resulting light curves were high-pass filtered using a triangular smoothing of width one day, to minimize the effects of the long-period instrumental drifts. The power spectra were produced from a single source using the Lomb-Scargle periodogram (Scargle 1982), which had been carefully calibrated to comply with Parseval's theorem (see Appourchaux 2011).

Kepler observations are divided into three-month-long Quarters (Q). We selected a subset of 42 cool main-sequence stars and subgiants observed during quarters Q5, Q6, and Q7 (March 22, 2010 to December 22, 2010) because they had oscillation modes with high signal-to-noise ratios ranging from 1.8 to 50 in the power spectrum. The frequency resolution is about $0.04 \mu \mathrm{Hz}$. Figure 1 shows the measured large frequency separation of these 42 stars as a function of their effective temperature provided by Pinsonneault et al. (2011). The large separation is derived from individual mode frequencies at $v_{\max }$ from the All data set (see Table 1). We took care to analyse solartype stars without avoided crossings, since these may reduce the observed linewidths. The avoided crossings were detected by visual inspection of the echelle diagram; examples of such avoided crossings in other stars can be found in Deheuvels et al. (2010), Metcalfe et al. (2010), Mathur et al. (2011), Campante et al. (2011), and Bedding (2011).

\subsection{Mode parameter extraction}

The mode parameter extraction was performed by 11 fitters. The list of fitted modes were compared for completeness and five fitting methods were selected to finalise the parameters: two fitters (IAS, BIR), that applied maximum likelihood estimators (MLE), and three Bayesian fitters (SYD, MAR, and AAU).

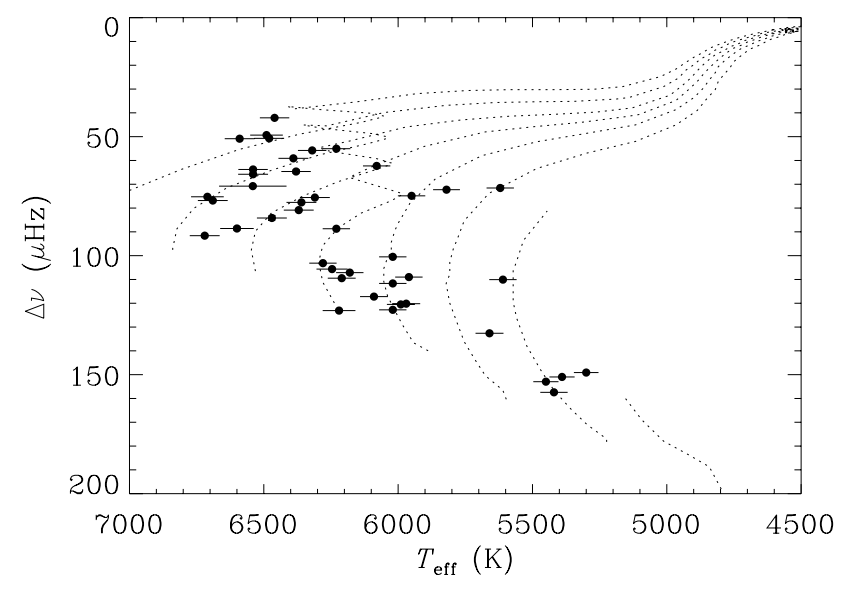

Fig. 1. Large separation as a function of effective temperature of the stars used in this study. The error bars on the large separations are within the thickness of the symbol. The evolutionary tracks for stars of mass from $0.8 M_{\odot}$ (most right) to $1.5 M_{\odot}$ (most left) (by step of $0.1 M_{\odot}$ ) are shown as dotted lines. The tracks are derived from Marigo et al. (2008).

The power spectra were modelled over a frequency range covering typically about 15 to 20 large separations $(=\Delta v)$. For each radial order, the model parameters were mode frequencies (one each for $l=0,1,2$ ), a single mode height (with an assumed ratio of $H_{1} / H_{0}=1.5, H_{2} / H_{0}=0.5$ ), and a single mode linewidth. In the case of AAU only, the $l=0$ linewidths were fitted and the linewidths of the other degrees were interpolated inbetween two $l=0$ mode linewidths. The relative heights $H_{(l, m)}$ of the split components of the modes depend on the stellar inclination angle as given by Gizon \& Solanki (2003). For each star, the rotational splitting and stellar inclination angle were chosen to be common across all the modes. The mode profile was assumed to be Lorentzian. The background was modelled using a multi-component Harvey model (Harvey 1985) with two parameters and a white noise component. We used a single Harvey component for all stars, and a double component for 11 stars (BIR's stars). In total, the number of free parameters for 15 orders was at least $5 \times 15+2=77$.

The two models described above were used to fit the parameters of the stars using MLE. All 42 stars were fitted by IAS, 16 of which were fitted by IAS alone. Eleven stars were fitted by BIR. The fit was done without and with rotational splitting; the significance of the splitting and angle was then tested using the likelihood ratio test, by applying the $\mathrm{H}_{0}$ hypothesis with a cutoff for a $\chi^{2}$ with 2 d.o.f of $\Delta \log ($ likelihood $)=9.2$ (4.6) or a probability of $10^{-4}\left(10^{-2}\right)$ for IAS, and for BIR, respectively. Formal uncertainties in each parameter were derived from the inverse of the Hessian matrix (for more details about MLE, significance, and formal errors, see Appourchaux 2011).

Fifteen stars with large mode linewidths were fitted with a Bayesian approach using different sampling methods. SYD and AAU employed MCMC (Benomar et al. 2009; Handberg \& Campante 2011), while MAR used nested sampling via the code MultiNest (Feroz et al. 2009). For the nested sampling approach, the large number of parameters forced us to use MultiNest's constant efficiency, mono-modal mode. The priors on the central frequency and inclination angle were uniform. The prior on the splitting was either uniform from $0-10 \mu \mathrm{Hz}$ (MAR) or a combination of a uniform prior over $0-2 \mu \mathrm{Hz}$ and a decaying Gaussian (SYD, AAU). The priors on mode height were modified Jeffreys priors (Benomar et al. 2009; Gruberbauer et al. 2009), and the priors on the linewidth were either uniform 
Table 1. Data set of fitted stars.

\begin{tabular}{lcccc}
\hline \hline Dataset & Fitter & Method & \# of stars & Comment \\
\hline I & IAS & MLE & 16 & No common stars \\
II & BIR & MLE & 11 & No common stars \\
III & SYD & Bayes & 7 & Common stars \\
IV & MAR & Bayes & 7 & Common stars $^{\dagger}$ \\
V & AAU & Bayes & 7 & Common stars $^{\dagger}$ \\
All & IAS & MLE & 42 & All stars included \\
\hline
\end{tabular}

Notes. ${ }^{(\dagger)}$ From these, 3 stars commonly fitted by SYD, MAR and AAU.

(MAR) or modified Jeffreys priors (SYD, AAU). The error bars were derived from the marginal posterior distribution of each parameter. Each Bayesian fitter had seven stars to fit: four stars + three common stars. The latter wer used to compare with the Bayesian methods. Priors on frequencies were set after visual inspection of the power spectrum. Modes of degree $l=2$ were assumed to be on the low-frequency side of the $l=0$ (i.e., the small spacing $d_{02}$ was assumed positive). To avoid spurious results, one of the Bayesian fitters (SYD) also used a smoothness condition on the frequency for each degree.

The different data sets available are summarized in Table 1.

\subsection{Linewidths}

In a similar fashion to Baudin et al. (2011a), we derived the mean linewidth $\left(\Gamma_{v_{\max }}\right)$ at maximum mode height and at maximum mode amplitude by taking the weighted average of three linewidths of three orders around the frequency of these maxima (see Tables 4 and 5) The derivation of $\Gamma_{v_{\max }}$ is rather immune to systematic effects resulting from the three-mode average because at these frequencies the observed linewidths exhibit a plateau, as shown theoretically by Houdek et al. (1999) and observed in the Sun by Fröhlich et al. (1995).

Individual mode linewidths can have systematic errors resulting from the incorrect estimation of several mode profile parameters. In addition, an over- or underestimation of mode linewidths will provide an under- or overestimation of mode heights, respectively. Estimates of these systematic errors can be derived using the procedure developed by Toutain et al. (2005), which consists of fitting one model profile, without using MonteCarlo simulations.

The main parameters producing systematic errors in the mode linewidths are: the background noise $B$, the mode height ratio, and the splitting.

The major source of systematic errors on mode height and mode linewidth is the biased estimation of the background noise. An estimate of the mode linewidth bias can be derived for a single mode using the analytical formulae provided by Toutain \& Appourchaux (1994). We can then derive the bias in the mode linewidth as a function of the error in $\Delta B$ and the inverse signalto-noise ratio $(\beta=B / H)$ in the power spectrum as

$\frac{\Delta \Gamma}{\Gamma}=k(\beta, \Gamma, \Delta v) \frac{\Delta B}{B}$,

where $\Delta v$ is the window over which the fit is performed for that single mode. Typically $k$ is negative and of order 1 , i.e., an under-estimation of the background by $10 \%$ will lead to an over-estimation of the linewidth by $10 \%$. Another source of systematic errors is the assumption that the ratios of mode height be fixed to some given values. There is indeed a variation in the mode height ratios with effective temperature as shown by
Table 2. Parameters of the fit of Eq. (2) and their random errors in linewidth measured at maximum mode height.

\begin{tabular}{lcccc}
\hline \hline Dataset & $T_{\text {eff }}$ & $\Gamma_{0}(\mu \mathrm{Hz})$ & $\alpha(\mu \mathrm{Hz})$ & $\mathrm{s}$ \\
\hline I+II+III+IV+V $^{+}$ & Pins. & $0.35 \pm 0.13$ & $0.91 \pm 0.16$ & $13.7 \pm 1.4$ \\
I+II $^{\dagger}$ & Pins. & $0.32 \pm 0.17$ & $0.93 \pm 0.20$ & $12.7 \pm 2.1$ \\
All & Pins. & $0.46 \pm 0.09$ & $0.75 \pm 0.11$ & $15.4 \pm 1.3$ \\
\hline I+II+III+IV+V & Casa. & $0.20 \pm 0.14$ & $0.97 \pm 0.17$ & $13.0 \pm 1.4$ \\
\hline
\end{tabular}

Notes. ${ }^{(\dagger)}$ Range for these stars is $5300 \mathrm{~K}$ to $6400 \mathrm{~K}$.

Ballot et al. (2011). The resulting underestimation of these ratios is typically no larger than 0.1 , which corresponds roughly to an underestimation of the linewidths of no larger than $3 \%$. A minor source of systematic errors comes from the rotational splitting. In the case for which the splitting is not detected (typically when the splitting is no greater than $10 \%$ of the linewidth), the linewidth will be overestimated by about $6 \%$ for $\Gamma=10 \mu \mathrm{Hz}$, and by about $3 \%$ for $\Gamma=3 \mu \mathrm{Hz}$. When the splitting is larger, there is no correlation between the detected splitting and the linewidth (Toutain \& Appourchaux 1994). All these values were either confirmed or inferred with the procedure suggested by Toutain et al. (2005).

Last but not least, an extrinsic systematic effect on the linewidth is caused by stellar activity. It was shown by Chaplin et al. (2000), that the solar linewidth may change by typically $20 \%$ at the location of the dip. We are aware that this can have an effect on the mean linewidth reported here. For many stars, this effect cannot be assessed with such a short observation duration of nine months.

\section{Discussion}

Figure 2 shows the linewidth measured at a maximum mode height as a function of effective temperature. We note that Chaplin et al. (2009) proposed a scaling relation, which provides a variation in the mode linewidth by a factor of 2.7 between $6800 \mathrm{~K}$ and $5300 \mathrm{~K}$; while Baudin et al. (2011b) provides a factor of 53.9 for the same temperature change. The measured ratio, here, is closer to 10 . It is clear that neither dependence is adequate to explain our measurements. The results of Chaplin et al. (2009) were based on predicted mode lifetimes from pulsation computations, and also on a small number of relatively short ground-based observations, which are potentially subject to large systematic errors.

We tested three forms of the $T_{\text {eff }}$ relations, namely an exponential variation, a pure power law, and a power law with a flat component. Without any physical basis for choosing between the different relations, we adopted the one with the lowest $\chi^{2}$, which was the third of these

$\Gamma=\Gamma_{0}+\alpha\left(\frac{T_{\mathrm{eff}}}{5777}\right)^{s}$.

The effective temperatures were derived from two re-calibrations of the photometry in the Kepler input catalog: one based on the griz photometry (Pinsonneault et al. 2011) and the other on the application of the infrared flux method using 2MASS JHK data (Casagrande et al. 2010, 2006). The random errors in the fitted parameters were derived using Monte Carlo simulations of the fit taking into account random errors on both the effective temperature and the linewidths.

Tables 2 and 3 give the results of the fitted parameters of the linewidth at $v_{\max }$ for the two different effective temperatures and 


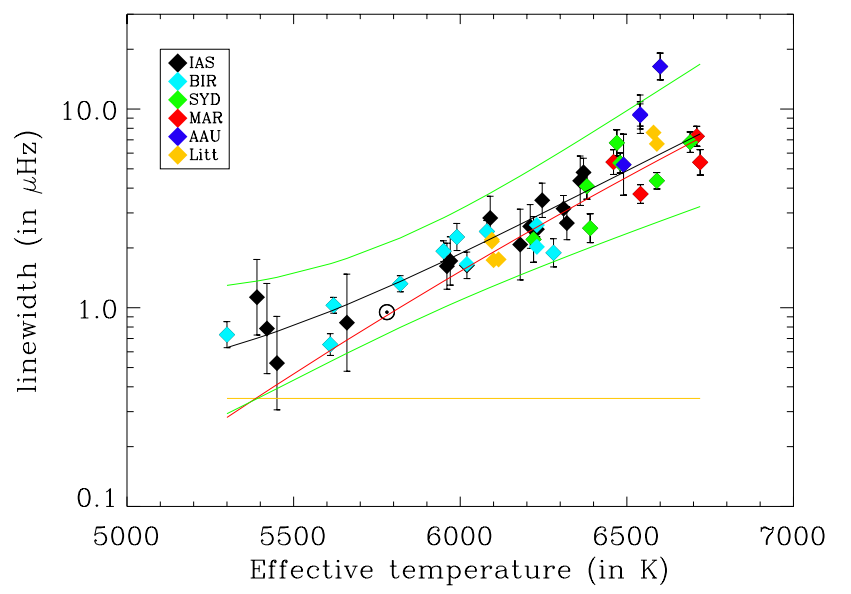

Fig. 2. Average mode linewidth at maximum mode height (and their 3- $\sigma$ error bars) as a function of effective temperature (provided by Pinsonneault et al. 2011). The error bars in the effective temperatures, although not shown here, are indeed included in the error analysis. Average mode linewidth fitted by IAS (black), by BIR (cyan), by SYD ange). Fitted average linewidth (black line). The 3- $\sigma$ error bars in the fitted average linewidth (green lines). Power-law component of the fit (red line). Flat component at low $T_{\text {eff }}$ (orange line). The mean mode linewidth of the Sun is indicated at $5777 \mathrm{~K}$.

Table 3. Parameters of the fit of Eq. (2) and their random errors bars in linewidth measured at maximum mode amplitude.

\begin{tabular}{lcccc}
\hline \hline Dataset & $T_{\text {eff }}$ & $\Gamma_{0}(\mu \mathrm{Hz})$ & $\alpha(\mu \mathrm{Hz})$ & $s$ \\
\hline I+II+III+IV+V & Pins. & $0.64 \pm 0.11$ & $0.66 \pm 0.14$ & $16.7 \pm 1.8$ \\
I+II $^{\dagger}$ & Pins. & $0.65 \pm 0.10$ & $0.64 \pm 0.13$ & $16.1 \pm 2.3$ \\
All & Pins. & $0.65 \pm 0.09$ & $0.64 \pm 0.10$ & $17.0 \pm 1.4$ \\
\hline I+II+III+IV+V & Casa. & $0.49 \pm 0.12$ & $0.75 \pm 0.15$ & $15.5 \pm 1.6$ \\
\hline
\end{tabular}

Notes. ${ }^{(\dagger)}$ Range for these stars is $5300 \mathrm{~K}$ to $6400 \mathrm{~K}$.

the two different ways of measuring $v_{\max }$. This latter can be derived either from the maximum of the mode amplitude, which is $\propto E$ (where $\sqrt{E}$ is the energy injected by convection), or from the maximum of mode height, which is $\propto E / \Gamma$. We used five different sets of linewidth data to study the impact of the different method upon the fitted parameters: all fitted linewidth (MLE and Bayesian), all fitted linewidth (excluding either BIR or IAS's), MLE (fitted by IAS and BIR only), and MLE (fitted only by IAS).

Here we note that the power-law indices are rather close to the index given by Baudin et al. (2011b) (see Table 2). The mode linewidth measured at the maximum mode height is systematically lower on average by about $10 \%$ than that measured at the maximum amplitude. This is because the frequency of maximum amplitude tends to be higher than the frequency of maximum mode height.

The different power-law index between the two sources of effective temperature is mainly due to the range of temperature being smaller for Pinsonneault et al. (2011) than Casagrande et al. (2010); the reduction is $75 \mathrm{~K}$, mainly at the high temperatures. The lower temperature range would increase $s$ by 1.0 and 1.5 , which is roughly in agreement with Tables 2 and 3, respectively.

We also studied the impact of having different fitters on the derived parameters. From Tables 2 and 3, we can see that the fitted parameters were the same within the error bars when we combined the MLE fits with the Bayesian fits. There was a much (green), by MAR (red), by AAU (blue), from Baudin et al. (2011a) (or-

Table 4. Natural logarithm of the linewidth measured at maximum mode height with their error bars for each star, together with the frequency of the maximum, the effective temperature of Pinsonneault et al. (2011), and of Casagrande et al. (2010, 2006), with their respective error bars.

\begin{tabular}{|c|c|c|c|c|c|}
\hline KIC number & $T_{\text {eff }}^{\text {Pins }}$ & $T_{\mathrm{eff}}^{\mathrm{Cas}}$ & $v_{\max }$ & $\gamma(\ln \mu \mathrm{Hz})$ & Fitter \\
\hline 1435467 & $6541 \pm 126$ & $6433 \pm 58$ & 1414.3 & $1.422 \pm 0.073$ & IAS \\
\hline 2837475 & $6710 \pm 61$ & $6664 \pm 92$ & 1585.3 & $2.228 \pm 0.072$ & IAS \\
\hline 3424541 & $6460 \pm 55$ & $6723 \pm 83$ & 678.8 & $1.480 \pm 0.112$ & IAS \\
\hline 3427720 & $5970 \pm 52$ & $6100 \pm 80$ & 2684.6 & $0.542 \pm 0.093$ & IAS \\
\hline 3733735 & $6720 \pm 56$ & $6827 \pm 96$ & 2026.9 & $2.227 \pm 0.102$ & IAS \\
\hline 3735871 & $6220 \pm 61$ & $6298 \pm 67$ & 2747.4 & $1.012 \pm 0.137$ & IAS \\
\hline 6116048 & $6020 \pm 51$ & $6073 \pm 69$ & 2150.0 & $0.420 \pm 0.072$ & IAS \\
\hline 6508366 & $6480 \pm 56$ & $6379 \pm 90$ & 979.8 & $1.599 \pm 0.074$ & IAS \\
\hline 6603624 & $5610 \pm 51$ & $5672 \pm 58$ & 2367.0 & $-0.423 \pm 0.078$ & IAS \\
\hline 6679371 & $6590 \pm 56$ & $6473 \pm 89$ & 854.0 & $1.623 \pm 0.062$ & IAS \\
\hline 6933899 & $5820 \pm 50$ & $5837 \pm 73$ & 1393.9 & $0.239 \pm 0.065$ & IAS \\
\hline 7103006 & $6390 \pm 56$ & $6381 \pm 84$ & 1132.8 & $1.415 \pm 0.080$ & IAS \\
\hline 7106245 & $6020 \pm 51$ & $6041 \pm 69$ & 2382.8 & $0.312 \pm 0.182$ & IAS \\
\hline 7206837 & $6360 \pm 56$ & $6428 \pm 75$ & 1509.0 & $1.472 \pm 0.095$ & IAS \\
\hline 7871531 & $5390 \pm 47$ & $5331 \pm 42$ & 3254.7 & $0.122 \pm 0.146$ & IAS \\
\hline 8006161 & $5300 \pm 46$ & $5399 \pm 41$ & 3518.5 & $-0.354 \pm 0.085$ & IAS \\
\hline 8228742 & $6080 \pm 51$ & $6235 \pm 76$ & 1126.9 & $0.824 \pm 0.070$ & IAS \\
\hline 8379927 & $5990 \pm 52$ & $5965 \pm 62$ & 2684.0 & $0.815 \pm 0.066$ & IAS \\
\hline 8394589 & $6210 \pm 52$ & $6276 \pm 75$ & 2328.6 & $0.942 \pm 0.085$ & IAS \\
\hline 8694723 & $6310 \pm 56$ & $6401 \pm 73$ & 1435.3 & $1.148 \pm 0.051$ & IAS \\
\hline 9025370 & $5660 \pm 52$ & $5737 \pm 69$ & 2848.3 & $-0.173 \pm 0.188$ & IAS \\
\hline 9098294 & $5960 \pm 51$ & $5984 \pm 60$ & 2334.9 & $0.481 \pm 0.089$ & IAS \\
\hline 9139151 & $6090 \pm 52$ & $6226 \pm 78$ & 2620.2 & $1.040 \pm 0.084$ & IAS \\
\hline 9139163 & $6370 \pm 56$ & $6510 \pm 90$ & 1704.5 & $1.569 \pm 0.055$ & IAS \\
\hline 9206432 & $6470 \pm 56$ & $6677 \pm 109$ & 1903.9 & $2.129 \pm 0.086$ & IAS \\
\hline 9410862 & $6180 \pm 51$ & $6174 \pm 65$ & 2184.9 & $0.732 \pm 0.137$ & IAS \\
\hline 9812850 & $6380 \pm 55$ & $6382 \pm 95$ & 1264.4 & $1.680 \pm 0.078$ & IAS \\
\hline 9955598 & $5450 \pm 47$ & $5492 \pm 45$ & 3453.4 & $-0.642 \pm 0.180$ & IAS \\
\hline 10018963 & $6230 \pm 52$ & $6154 \pm 78$ & 947.2 & $0.854 \pm 0.052$ & IAS \\
\hline 10162436 & $6320 \pm 53$ & $6253 \pm 77$ & 1008.6 & $0.981 \pm 0.064$ & IAS \\
\hline 10355856 & $6540 \pm 56$ & $6595 \pm 77$ & 1280.5 & $1.754 \pm 0.079$ & IAS \\
\hline 10454113 & $6246 \pm 58$ & $6071 \pm 74$ & 2333.2 & $1.245 \pm 0.066$ & IAS \\
\hline 10644253 & $6020 \pm 51$ & $6122 \pm 69$ & 2993.2 & $0.805 \pm 0.137$ & IAS \\
\hline 10909629 & $6490 \pm 61$ & $6420 \pm 73$ & 893.1 & $1.220 \pm 0.101$ & IAS \\
\hline 10963065 & $6280 \pm 51$ & $6177 \pm 67$ & 2195.5 & $0.822 \pm 0.064$ & IAS \\
\hline 11081729 & $6600 \pm 62$ & $6696 \pm 81$ & 1803.2 & $1.887 \pm 0.103$ & IAS \\
\hline 11244118 & $5620 \pm 51$ & $5824 \pm 62$ & 1383.7 & $-0.081 \pm 0.077$ & IAS \\
\hline 11253226 & $6690 \pm 56$ & $6789 \pm 99$ & 1685.8 & $2.166 \pm 0.056$ & IAS \\
\hline 11772920 & $5420 \pm 51$ & $5440 \pm 44$ & 3394.7 & $-0.241 \pm 0.174$ & IAS \\
\hline 12009504 & $6230 \pm 51$ & $6337 \pm 71$ & 1870.5 & $0.628 \pm 0.092$ & IAS \\
\hline 12258514 & $5950 \pm 51$ & $5967 \pm 70$ & 1517.1 & $0.515 \pm 0.053$ & IAS \\
\hline 12317678 & $6540 \pm 55$ & $6558 \pm 86$ & 1201.9 & $1.594 \pm 0.058$ & IAS \\
\hline 6116048 & $6020 \pm 51$ & $6073 \pm 69$ & 2150.0 & $0.507 \pm 0.053$ & BIR \\
\hline 6603624 & $5610 \pm 51$ & $5672 \pm 58$ & 2367.0 & $-0.427 \pm 0.042$ & BIR \\
\hline 6933899 & $5820 \pm 50$ & $5837 \pm 73$ & 1321.5 & $0.278 \pm 0.031$ & BIR \\
\hline 8006161 & $5300 \pm 46$ & $5399 \pm 41$ & 3518.4 & $-0.312 \pm 0.050$ & BIR \\
\hline 8228742 & $6080 \pm 51$ & $6235 \pm 76$ & 1189.0 & $0.884 \pm 0.042$ & BIR \\
\hline 8379927 & $5990 \pm 52$ & $5965 \pm 62$ & 2684.0 & $0.820 \pm 0.052$ & BIR \\
\hline 10018963 & $6230 \pm 52$ & $6154 \pm 78$ & 947.2 & $0.958 \pm 0.033$ & BIR \\
\hline 10963065 & $6280 \pm 51$ & $6177 \pm 67$ & 2092.4 & $0.637 \pm 0.054$ & BIR \\
\hline 11244118 & $5620 \pm 51$ & $5824 \pm 62$ & 1312.2 & $0.028 \pm 0.031$ & BIR \\
\hline 12009504 & $6230 \pm 51$ & $6337 \pm 71$ & 1870.5 & $0.706 \pm 0.069$ & BIR \\
\hline 12258514 & $5950 \pm 51$ & $5967 \pm 70$ & 1517.1 & $0.656 \pm 0.040$ & BIR \\
\hline 3735871 & $6220 \pm 61$ & $6298 \pm 67$ & 2747.3 & $0.792 \pm 0.088$ & SYD \\
\hline 6508366 & $6480 \pm 56$ & $6379 \pm 90$ & 980.0 & $1.678 \pm 0.039$ & SYD \\
\hline 6679371 & $6590 \pm 56$ & $6473 \pm 89$ & 854.0 & $1.472 \pm 0.032$ & SYD \\
\hline 7103006 & $6390 \pm 56$ & $6381 \pm 84$ & 1133.1 & $0.922 \pm 0.056$ & SYD \\
\hline 9206432 & $6470 \pm 56$ & $6677 \pm 109$ & 1864.6 & $1.911 \pm 0.051$ & SYD \\
\hline 9812850 & $6380 \pm 55$ & $6382 \pm 95$ & 1170.3 & $1.408 \pm 0.050$ & SYD \\
\hline 11253226 & $6690 \pm 56$ & $6789 \pm 99$ & 1608.9 & $1.918 \pm 0.040$ & SYD \\
\hline 1435467 & $6541 \pm 126$ & $6789 \pm 99$ & 1205.4 & $1.318 \pm 0.036$ & MAR \\
\hline 2837475 & $6710 \pm 61$ & $6433 \pm 58$ & 1509.2 & $1.987 \pm 0.039$ & MAR \\
\hline 3424541 & $6460 \pm 55$ & $6664 \pm 92$ & 677.8 & $1.688 \pm 0.048$ & MAR \\
\hline 3733735 & $6720 \pm 56$ & $6723 \pm 83$ & 1655.4 & $1.685 \pm 0.049$ & MAR \\
\hline 10355856 & $6540 \pm 56$ & $6595 \pm 77$ & 1280.4 & $2.242 \pm 0.074$ & AAU \\
\hline 10909629 & $6490 \pm 61$ & $6420 \pm 73$ & 942.5 & $1.657 \pm 0.118$ & AAU \\
\hline 11081729 & $6600 \pm 62$ & $6696 \pm 81$ & 1892.2 & $2.796 \pm 0.052$ & AAU \\
\hline 12317678 & $6540 \pm 55$ & $6789 \pm 99$ & 1166.8 & $2.229 \pm 0.053$ & AAU \\
\hline
\end{tabular}


Table 5. Natural logarithm of the linewidth measured at maximum amplitude with their error bars for each star, together with the frequency of the maximum, the effective temperature of Pinsonneault et al. (2011), and of Casagrande et al. (2010, 2006), with their respective error bars.

\begin{tabular}{|c|c|c|c|c|c|}
\hline KIC number & $T_{\mathrm{eff}}^{\text {Pins }}$ & $T_{\mathrm{eff}}^{\mathrm{Cas}}$ & $v_{\max }$ & $\gamma(\ln \mu \mathrm{Hz})$ & Fitter \\
\hline 1435467 & $6541 \pm 126$ & $6433 \pm 58$ & 1344.1 & $1.462 \pm 0.074$ & IAS \\
\hline 2837475 & $6710 \pm 61$ & $6664 \pm 92$ & 1660.2 & $2.292 \pm 0.065$ & IAS \\
\hline 3424541 & $6460 \pm 55$ & $6723 \pm 83$ & 841.9 & $1.971 \pm 0.119$ & IAS \\
\hline 3427720 & $5970 \pm 52$ & $6100 \pm 80$ & 2684.6 & $0.542 \pm 0.093$ & IAS \\
\hline 3733735 & $6720 \pm 56$ & $6827 \pm 96$ & 2119.0 & $2.286 \pm 0.099$ & IAS \\
\hline 3735871 & $6220 \pm 61$ & $6298 \pm 67$ & 2747.4 & $1.012 \pm 0.137$ & IAS \\
\hline 6116048 & $6020 \pm 51$ & $6073 \pm 69$ & 2150.0 & $0.420 \pm 0.072$ & IAS \\
\hline 6508366 & $6480 \pm 56$ & $6379 \pm 90$ & 979.8 & $1.599 \pm 0.074$ & IAS \\
\hline 6603624 & $5610 \pm 51$ & $5672 \pm 58$ & 2367.0 & $-0.423 \pm 0.078$ & IAS \\
\hline 6679371 & $6590 \pm 56$ & $6473 \pm 89$ & 1006.6 & $1.851 \pm 0.061$ & IAS \\
\hline 6933899 & $5820 \pm 50$ & $5837 \pm 73$ & 1393.9 & $0.239 \pm 0.065$ & IAS \\
\hline 7103006 & $6390 \pm 56$ & $6381 \pm 84$ & 1251.7 & $1.708 \pm 0.074$ & IAS \\
\hline 7106245 & $6020 \pm 51$ & $6041 \pm 69$ & 2382.8 & $0.312 \pm 0.182$ & IAS \\
\hline 7206837 & $6360 \pm 56$ & $6428 \pm 75$ & 1745.1 & $1.547 \pm 0.095$ & IAS \\
\hline 7871531 & $5390 \pm 47$ & $5331 \pm 42$ & 3254.7 & $0.122 \pm 0.146$ & IAS \\
\hline 8006161 & $5300 \pm 46$ & $5399 \pm 41$ & 3667.8 & $-0.110 \pm 0.082$ & IAS \\
\hline 8228742 & $6080 \pm 51$ & $6235 \pm 76$ & 1251.5 & $0.881 \pm 0.066$ & IAS \\
\hline 8379927 & $5990 \pm 52$ & $5965 \pm 62$ & 2804.1 & $0.979 \pm 0.064$ & IAS \\
\hline 8394589 & $6210 \pm 52$ & $6276 \pm 75$ & 2437.9 & $1.178 \pm 0.087$ & IAS \\
\hline 8694723 & $6310 \pm 56$ & $6401 \pm 73$ & 1285.9 & $1.195 \pm 0.054$ & IAS \\
\hline 9025370 & $5660 \pm 52$ & $5737 \pm 69$ & 2981.0 & $0.055 \pm 0.161$ & IAS \\
\hline 9098294 & $5960 \pm 51$ & $5984 \pm 60$ & 2334.9 & $0.481 \pm 0.089$ & IAS \\
\hline 9139151 & $6090 \pm 52$ & $6226 \pm 78$ & 2620.2 & $1.04 \pm 0.084$ & IAS \\
\hline 9139163 & $6370 \pm 56$ & $6510 \pm 90$ & 1624.1 & $1.565 \pm 0.056$ & IAS \\
\hline 9206432 & $6470 \pm 56$ & $6677 \pm 109$ & 1820.0 & $2.076 \pm 0.068$ & IAS \\
\hline 9410862 & $6180 \pm 51$ & $6174 \pm 65$ & 2292.1 & $1.074 \pm 0.145$ & IAS \\
\hline 9812850 & $6380 \pm 55$ & $6382 \pm 95$ & 1264.4 & $1.680 \pm 0.078$ & IAS \\
\hline 9955598 & $5450 \pm 47$ & $5492 \pm 45$ & 3759.7 & $0.207 \pm 0.156$ & IAS \\
\hline 10018963 & $6230 \pm 52$ & $6154 \pm 78$ & 947.2 & $0.854 \pm 0.052$ & IAS \\
\hline 10162436 & $6320 \pm 53$ & $6253 \pm 77$ & 1008.6 & $0.981 \pm 0.064$ & IAS \\
\hline 10355856 & $6540 \pm 56$ & $6595 \pm 77$ & 1280.5 & $1.754 \pm 0.079$ & IAS \\
\hline 10454113 & $6246 \pm 58$ & $6071 \pm 74$ & 2333.2 & $1.245 \pm 0.066$ & IAS \\
\hline 10644253 & $6020 \pm 51$ & $6122 \pm 69$ & 2993.2 & $0.805 \pm 0.137$ & IAS \\
\hline 10909629 & $6490 \pm 61$ & $6420 \pm 73$ & 844.0 & $1.156 \pm 0.101$ & IAS \\
\hline 10963065 & $6280 \pm 51$ & $6177 \pm 67$ & 2195.5 & $0.822 \pm 0.064$ & IAS \\
\hline 11081729 & $6600 \pm 62$ & $6696 \pm 81$ & 1922.7 & $1.981 \pm 0.097$ & IAS \\
\hline 11244118 & $5620 \pm 51$ & $5824 \pm 62$ & 1383.7 & $-0.081 \pm 0.077$ & IAS \\
\hline 11253226 & $6690 \pm 56$ & $6789 \pm 99$ & 1685.8 & $2.166 \pm 0.056$ & IAS \\
\hline 11772920 & $5420 \pm 51$ & $5440 \pm 44$ & 3867.5 & $0.441 \pm 0.229$ & IAS \\
\hline 12009504 & $6230 \pm 51$ & $6337 \pm 71$ & 1870.5 & $0.628 \pm 0.092$ & IAS \\
\hline 12258514 & $5950 \pm 51$ & $5967 \pm 70$ & 1517.1 & $0.515 \pm 0.053$ & IAS \\
\hline 12317678 & $6540 \pm 55$ & $6558 \pm 86$ & 1265.4 & $1.700 \pm 0.056$ & IAS \\
\hline 6116048 & $6020 \pm 51$ & $6073 \pm 69$ & 2150.0 & $0.507 \pm 0.053$ & BIR \\
\hline 6603624 & $5610 \pm 51$ & $5672 \pm 58$ & 2367.0 & $-0.427 \pm 0.042$ & BIR \\
\hline 6933899 & $5820 \pm 50$ & $5837 \pm 73$ & 1321.5 & $0.278 \pm 0.031$ & BIR \\
\hline 8006161 & $5300 \pm 46$ & $5399 \pm 41$ & 3667.8 & $0.020 \pm 0.046$ & BIR \\
\hline 8228742 & $6080 \pm 51$ & $6235 \pm 76$ & 1189.0 & $0.884 \pm 0.042$ & BIR \\
\hline 8379927 & $5990 \pm 52$ & $5965 \pm 62$ & 2804.1 & $0.999 \pm 0.051$ & BIR \\
\hline 10018963 & $6230 \pm 52$ & $6154 \pm 78$ & 947.2 & $0.958 \pm 0.033$ & BIR \\
\hline 10963065 & $6280 \pm 51$ & $6177 \pm 67$ & 2195.5 & $0.804 \pm 0.053$ & BIR \\
\hline 11244118 & $5620 \pm 51$ & $5824 \pm 62$ & 1383.7 & $0.060 \pm 0.029$ & BIR \\
\hline 12009504 & $6230 \pm 51$ & $6337 \pm 71$ & 1870.5 & $0.706 \pm 0.069$ & BIR \\
\hline 12258514 & $5950 \pm 51$ & $5967 \pm 70$ & 1517.1 & $0.656 \pm 0.040$ & BIR \\
\hline 3735871 & $6220 \pm 61$ & $6298 \pm 67$ & 2870.1 & $0.812 \pm 0.081$ & SYD \\
\hline 6508366 & $6480 \pm 56$ & $6379 \pm 90$ & 980.0 & $1.678 \pm 0.039$ & SYD \\
\hline 6679371 & $6590 \pm 56$ & $6473 \pm 89$ & 956.3 & $1.66 \pm 0.031$ & SYD \\
\hline 7103006 & $6390 \pm 56$ & $6381 \pm 84$ & 1133.1 & $0.922 \pm 0.056$ & SYD \\
\hline 9206432 & $6470 \pm 56$ & $6677 \pm 109$ & 1864.6 & $1.911 \pm 0.051$ & SYD \\
\hline 9812850 & $6380 \pm 55$ & $6382 \pm 95$ & 1298.6 & $1.466 \pm 0.047$ & SYD \\
\hline 11253226 & $6690 \pm 56$ & $6789 \pm 99$ & 1685.4 & $1.997 \pm 0.038$ & SYD \\
\hline 1435467 & $6541 \pm 126$ & $6789 \pm 99$ & 1344.1 & $1.508 \pm 0.029$ & MAR \\
\hline 2837475 & $6710 \pm 61$ & $6433 \pm 58$ & 1735.4 & $2.156 \pm 0.035$ & MAR \\
\hline 3424541 & $6460 \pm 55$ & $6664 \pm 92$ & 761.1 & $1.854 \pm 0.047$ & MAR \\
\hline 3733735 & $6720 \pm 56$ & $6723 \pm 83$ & 2027.2 & $2.256 \pm 0.043$ & MAR \\
\hline 10355856 & $6540 \pm 56$ & $6595 \pm 77$ & 1346.9 & $2.218 \pm 0.074$ & AAU \\
\hline 10909629 & $6490 \pm 61$ & $6420 \pm 73$ & 843.9 & $1.729 \pm 0.117$ & AAU \\
\hline 11081729 & $6600 \pm 62$ & $6696 \pm 81$ & 1982.6 & $2.792 \pm 0.053$ & AAU \\
\hline 12317678 & $6540 \pm 55$ & $6789 \pm 99$ & 1230.9 & $2.179 \pm 0.055$ & AAU \\
\hline
\end{tabular}

larger difference when we used the linewidth derived for all stars by IAS only (the only homogenous data set), thereby also including the stars for which the effective temperature was higher than $6400 \mathrm{~K}$. For that homogenous data set, the linewidths measured at high effective temperature were systematically higher than those measured by the Bayesian fitters by up to $15 \%$. Typically, a change in the linewidth at the highest effective temperature of $1 \mu \mathrm{Hz}$ will increase $s$ by 1 . The sensitivity of the power law in$\operatorname{dex} s$ to the high effective temperatures also explains why the index did not vary much when other data sets obtained at lower effective temperature were included (the data sets I and II from the MLE fitters are at low effective temperature).

\section{Conclusion}

We have studied the dependence of linewidth at maximum mode height and amplitude on $T_{\text {eff }}$ for two sources of effective temperature. We have shown using nine months of Kepler observations of 42 stars that the mode linewidth at both maximum mode height or maximum amplitude follows a scaling relation based on effective temperature, which is a combination of a power law and a lower bound. We stress that this scaling relation is only valid for the cool main-sequence and subgiant stars, and does not have predictive power outside the temperature range of these stars.

Acknowledgements. The authors wish to thank the entire Kepler team, without whom these results would not be possible. Funding for this Discovery mission is provided by NASA's Science Mission Directorate. We also thank all funding councils and agencies that have supported the activities of KASC Working Group 1, as well as the International Space Science Institute (ISSI). This research was supported in part by the National Science Foundation under Grant No. NSF PHY05-51164. W.J.C., G.A.V., and Y.E. acknowledge financial support from the UK Science and Technology Facilities Council (STFC). R.A.G. and G.R.D. has received funding from the European Community's Seventh Framework Programme (FP7/2007-2013) under grant agreement No. 269194. M.G. received financial support from an NSERC Vanier scholarship. This work employed computational facilities provided by ACEnet, the regional high performance computing consortium for universities in Atlantic Canada. SH acknowledges funding from the Nederlandse Organisatie voor Wetenschappelijk Onderzoek (NWO). G.H. acknowledges support by the Austrian Science Fund (FWF) project P21205-N16. R.H. acknowledges computing support from the National Solar Observatory. D.S. acknowledges the financial support from the Centre National d'Etudes Spatiales (CNES).

\section{References}

Appourchaux, T. 2011, A crash course on data analysis in asteroseismology, XXIIth Winter school of the Canary Islands [arXiv: 1103.5352]

Baglin, A. 2006, The CoRoT mission, pre-launch status, stellar seismology and planet finding, ed. M. Fridlund, A. Baglin, J. Lochard, \& L.Conroy, ESA SP1306, ESA Publication Division, Noordwijk, The Netherlands

Ballot, J., Barban, C., \& van't Veer-Menneret, C. 2011, A\&A, 531, A124

Balmforth, N. J. 1992, MNRAS, 255, 603

Baudin, F., Barban, C., Belkacem, K., et al. 2011a, A\&A, 529, A84

Baudin, F., Barban, C., Belkacem, K., et al. 2011b, A\&A, 535, C1

Bedding, T. R. 2011, Solar-like Oscillations: An Observational Perspective, XXIIth Winter school of the Canary Islands [arXiv: 1107.1723]

Belkacem, K., Goupil, M. J., Dupret, M. A., et al. 2011, A\&A, 530, A142

Benomar, O., Appourchaux, T., \& Baudin, F. 2009, A\&A, 506, 15

Borucki, W. J., Koch, D., Jenkins, J., et al. 2009, Science, 325, 709

Campante, T. L., Handberg, R., Mathur, S., et al. 2011, A\&A, 534, A6

Casagrande, L., Portinari, L., \& Flynn, C. 2006, MNRAS, 373, 13

Casagrande, L., Ramírez, I., Meléndez, J., Bessell, M., \& Asplund, M. 2010, A\&A, 512, A54

Chaplin, W. J., Elsworth, Y., Isaak, G. R., Miller, B. A., \& New, R. 2000, MNRAS, 313, 32

Chaplin, W. J., Houdek, G., Karoff, C., Elsworth, Y., \& New, R. 2009, A\&A, 500, L21

Chaplin, W. J., Kjeldsen, H., Christensen-Dalsgaard, J., et al. 2011, Science, 332, 213 
A\&A 537, A134 (2012)

Deheuvels, S., Bruntt, H., Michel, E., et al. 2010, A\&A, 515, A87 Feroz, F., Hobson, M. P., \& Bridges, M. 2009, MNRAS, 398, 1601 Fröhlich, C., Romero, J., Roth, H., et al. 1995, Sol. Phys., 162, 101

García, R. A., Hekker, S., Stello, D., et al. 2011, MNRAS, 414, L6

Gilliland, R. L., Jenkins, J. M., Borucki, W. J., et al. 2010, ApJ, 713, L160

Gizon, L., \& Solanki, S. K. 2003, ApJ, 589, 1009

Gruberbauer, M., Kallinger, T., Weiss, W. W., \& Guenther, D. B. 2009, A\&A, 506,1043

Handberg, R., \& Campante, T. L. 2011, A\&A, 527, A56

Harvey, J. 1985, in Future missions in solar, heliospheric and space plasma physics, ESA SP-235, ed. E. Rolfe, \& B. Battrick, ESA Publications Division, Noordwijk, The Netherlands, 199

Houdek, G., Balmforth, N. J., Christensen-Dalsgaard, J., \& Gough, D. O. 1999 A\&A, 351,582
Jenkins, J. M., Caldwell, D. A., Chandrasekaran, H., et al. 2010, ApJ, 713, L87 Kjeldsen, H., \& Bedding, T. R. 1995, A\&A, 293, 87

Kjeldsen, H., \& Bedding, T. R. 2011, A\&A, 529, L8

Marigo, P., Girardi, L., Bressan, A., et al. 2008, A\&A, 482, 883

Mathur, S., Handberg, R., Campante, T. L., et al. 2011, ApJ, 733, 95

Metcalfe, T. S., Monteiro, M. J. P. F. G., Thompson, M. J., et al. 2010, ApJ, 723, 1583

Pinsonneault, M. H., An, D., Molenda-Żakowicz, J., et al. 2011, ApJS, submitted [arXiv: 1110.4456$]$

Samadi, R. 2009 [arXiv:0912.0817]

Scargle, J. D. 1982, ApJ, 263, 835

Toutain, T., \& Appourchaux, T. 1994, A\&A, 289, 649

Toutain, T., Elsworth, Y., \& Chaplin, W. J. 2005, A\&A, 433, 713 